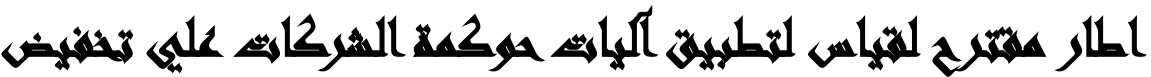

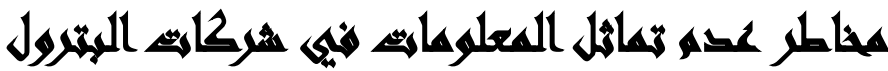

\section{كاسمة عالـ}

\section{$[r \mu]$}

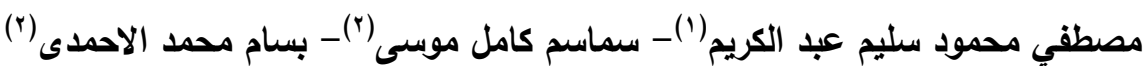

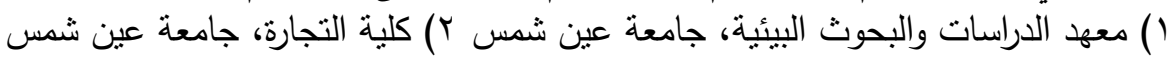

\section{المستخلص}

استهدف هذه الدراسة إلى التعرف على مدي تطبيق آليات حوكمة الثركات علي تخفيض مخاطر عدم تمانل المعلومات في شركات البترول، وذللك بتطبيق دراسة حالة علي التي

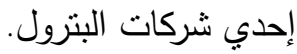

لتحقيق هذا الهدف إعتمد الباحثون على التأصيل النظري من خلال المنهج الإستقرائي،

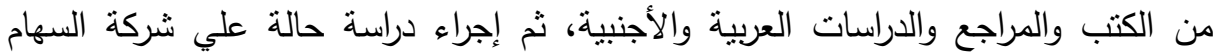

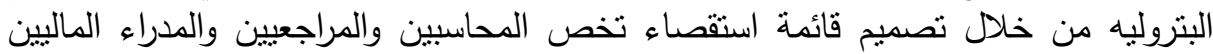

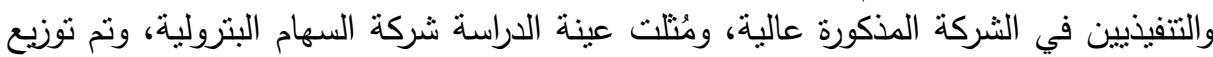

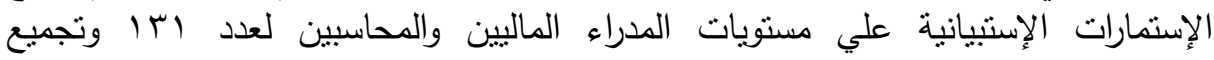

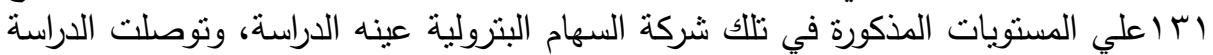

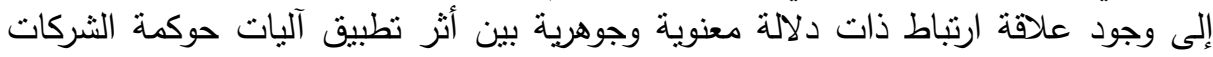

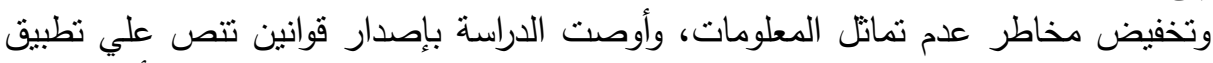

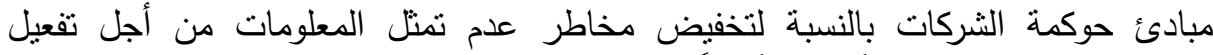

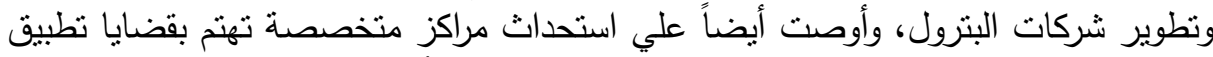

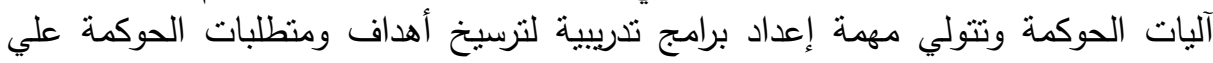
تخفيض مخاطر عدم تماثل المعلومات في الثركات.

\section{$x$}

لقد نالت حوكمة الثركات أهميه خاصه على مستوى كافه المؤسسات والمنظمات الاقليمية والدوليه وذلك بعد الأزمات المالية المختلفه التى حدثت فى كثير من الثركات

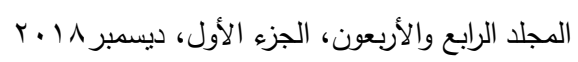


والمؤسسات وخاصة فى الدول المتقدمه فعلى سبيل المثال حدثت الإنهيارات المالية فى عدد

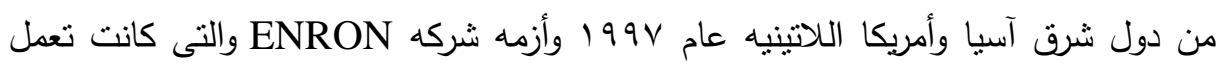

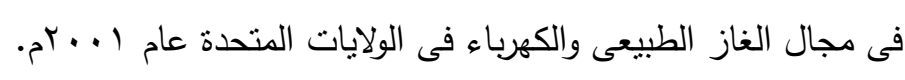

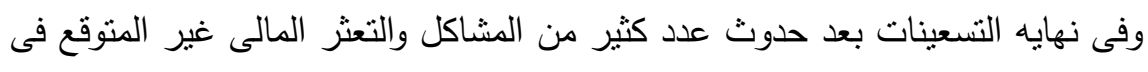
الولايات المتحده والمانبا وأيضا بنك بارينجز الإنجليزى، إضافة إلى أكتثاف الفئ المستثمرين

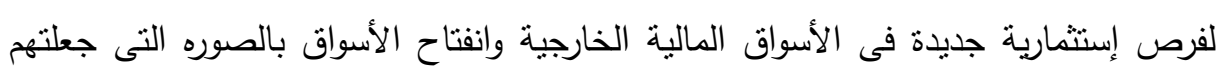

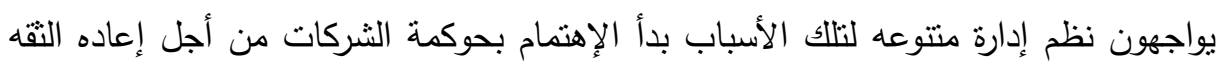

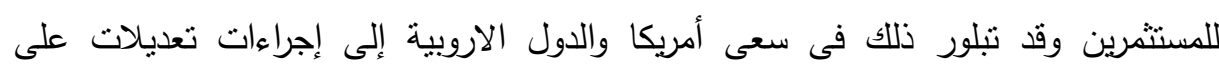

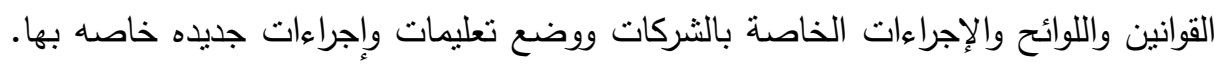

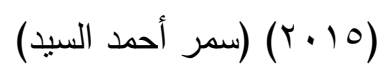

فالحوكمة تسعى دائما إلى تشجيع الإستتمار في الأسواق المالية من خلال تحقيق أفضل

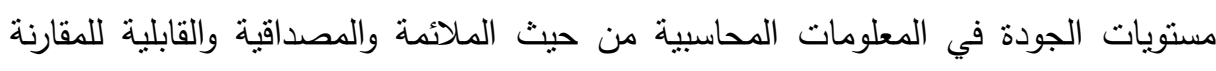
وزيادة الفهم والتفسير في ضوء إحتياجات بيئة الإستثمار، بالإضافة إلى تحقيق أفضل فئل

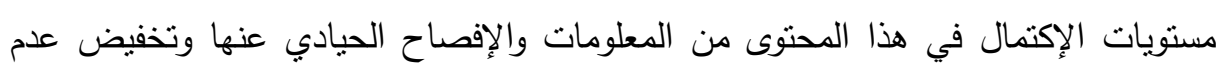

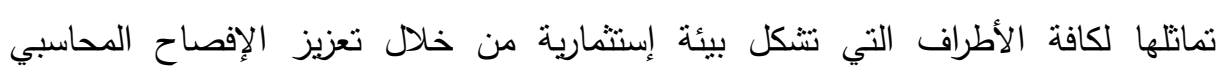
والثفافية فالمستثمرون يطلبون معلومات عن أعضاء المجلس والمديرين الخاصين المنفذين

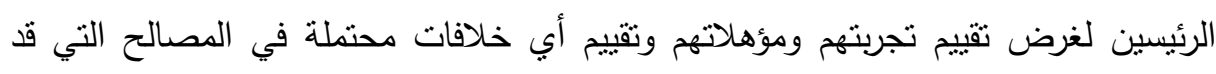

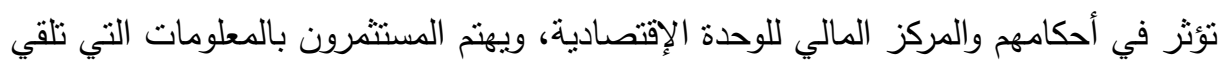

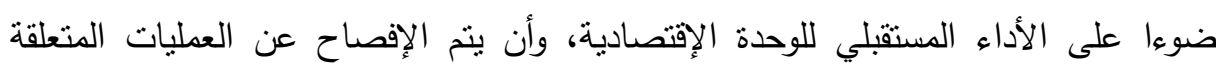

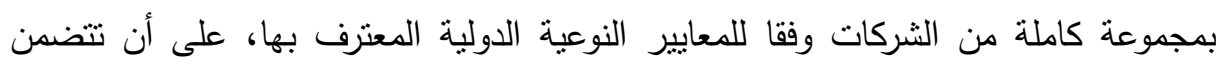

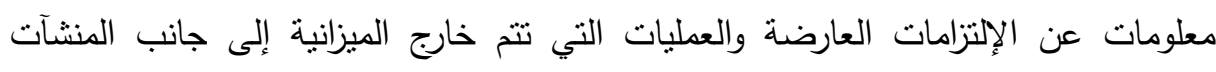

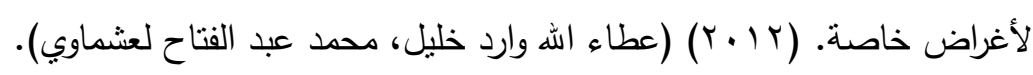




\section{And}

إن عدم تمانل المعلومات المحاسبية سيؤدي للإضرار بكفاءة السوق وقدرتها على نتجيع

الاستثمار، حيث أن كفاءة السوق لا تتحقق إلا في حالة تماتل المعلومات بين كافة الأطراف

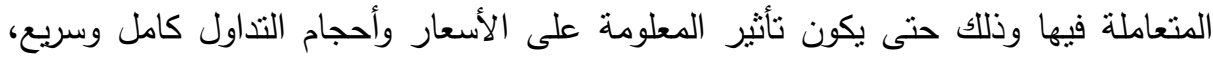

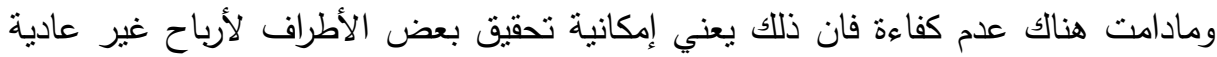

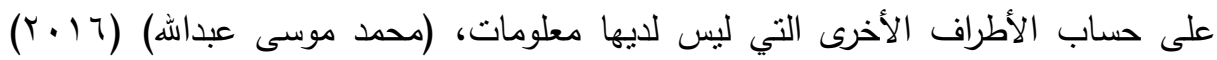
وتحقيق هذه الأرباح غير العادية سيؤدي بالطبع إلى الأضرار بالسوق، مما يترتب عليه

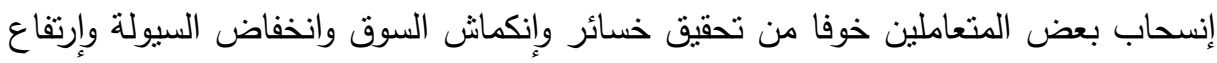
تكلفة المعاملات والتى سوف تؤثر سلباً على الاقتصاد القومي وعلى المستثرين وهم أكثر ونر

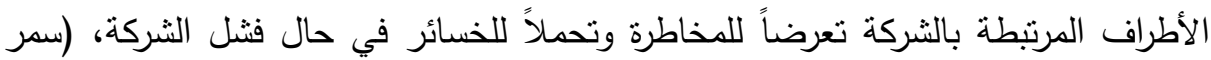

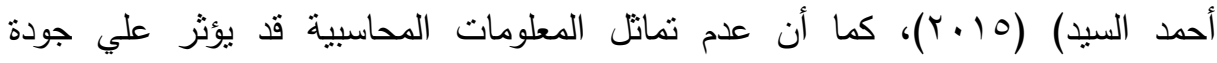

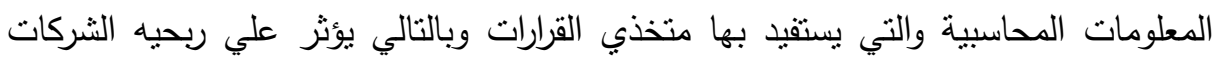
وعلي التتمية الإقتصادية عموماً، ويمكن صياغه مشكله البحث من خلال سؤال جوهري هل

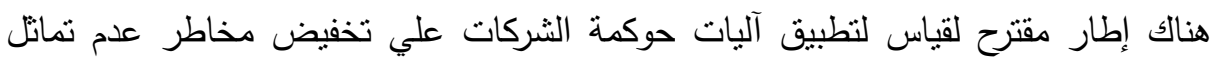

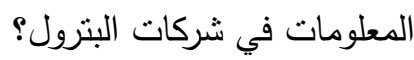

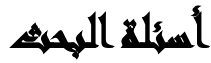

ويستمد من السؤال الرئيسي منه عدة أسئلة فرعية وهي: 1- ما إمكانية قياس التأثير البيئ لتطبيق آليات حوكمه الثركات؟ عنده

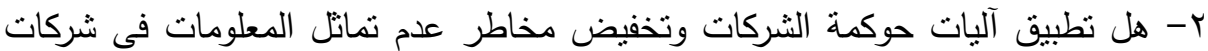

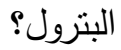

r- ما إمكانية قياس التأثير البيئ لتطبيق آليات حوكمة الثركه فى شركات البترول يؤدى إلى لي تخفيض مخاطر عدم تماتل المعلومات؟ 


\section{أهساهنغ التوراسما}

الهاف الرئيسي للاراسة: هو إطار مقترح لقياس لنطبيق آليات حوكمة الثركات علي تخفيض مخاطر عدم تماتل المعلومات في شركات البنرول، ويحقق هذا الهدف إلي إلقاء

$$
\text { الضوء علي مجموعة من أهداف فرعية وهي: }
$$

ا ـ مدي قياس التأثير البيئي لتطبيق آليات حوكمة الثركات؟

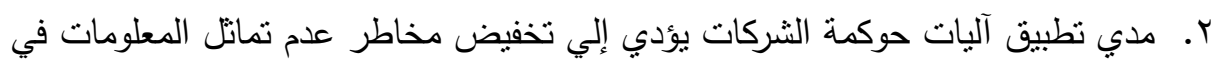

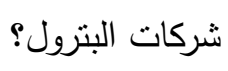

r. بمكن قياس الثأثير البيئي لتطبيق آليات حوكمة الثركة في شركات البنرول يؤدي إلي تخفيض مخاطر عدم تماثل المعلومات؟ المبات

\section{هزوضر الصواسما}

الفرض الأول: نوجد علاقة ذات دلاله إحصائية نحو أثز تطبيق آليات حوكمة الثركات وتخفيض مخاطر عدم تمانل المعلومات.

الفرض الثاني: توجد علاقة ذات دلاله إحصائية نحو أثز تطبيق آليات حوكمة الشركات علي تخفيض مخاطر عدم تماتل المعلومات والإفصاح المحاسبي في نثاط الثنركة.

\section{حصوض الكراسم}

اقتصرت الدراسة علي شركه السهام البتروليه وتقتصر فتزرة الدراسه على السنوات

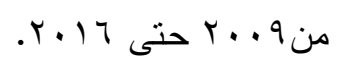

\section{منهمجية القراسلة}

تستمد هذه الدراسة أهميتها وتحقيقا لأهداف الدراسة سوف يقوم الباحث بالاعتماد علي

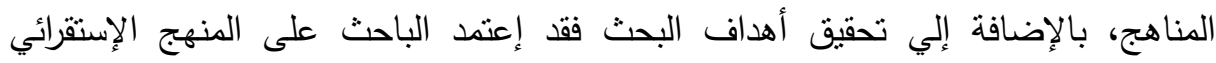
والمنهج الإننباطي وذلك علي النحو الاتي: الإني 
المنهج الإستقرائي: والذي يعتمد علي: من خلال استقراء بعض الكتابات والدراسات

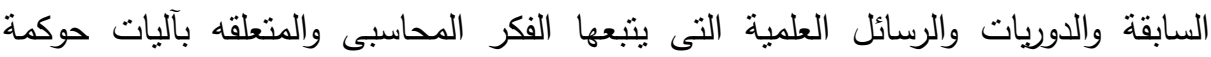
الثركات ومخاطر عدم تمانل المعلومات. المنهج التطبيقى: وذلك من خلال إتباع الباحث لمنهج دراسه الحاله بالنطبيق على إحدى شركات البترول بهدف اختيار فروض الدراسة والوصول إلى نتائج يمكن تعميمها على دلى دلى

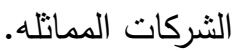

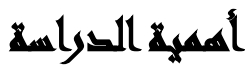

ترجع أهمية الدراسة من خلال أن أهية هذه الدراسة ترجع إلى نركيزها على جوده

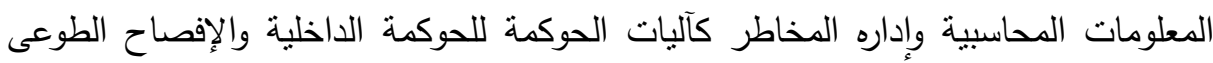
كآلية للحوكمة الخارجية على تعظيم قيمة حقوق المساهمين بهدف قياس جوده الحوكمة على مستوى الثركات.

\section{همطللحاهي التراسمة ( المهانهيه)}

1-مفهوم حوكمة الشركات: هي نتيجة للإنهيارات المالية لكبريات الثركات العالمية، وبعض الأحداث الاقتصادية كالأزمات وانتشار الفساد المالي والإداري في الشركات العالمية منل شركة "Enron"، هذا ما دفع بعض الكتاب والهيئات الدولية إلي البحث فيما

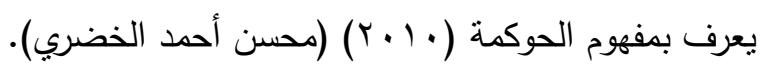

r-الإفصاح والثفافية: هو بيتم من خلال الإفصاح عن الأهداف المالية بدقة، نشر التقرير السنوي في موعده نشر التقارير المالية السنوية البيئية في الوقت المناسب، الإفصاح العادل عن النتائج السنوية نطبيق معايير المحاسبة والمراجعة، نوفير إمكانية

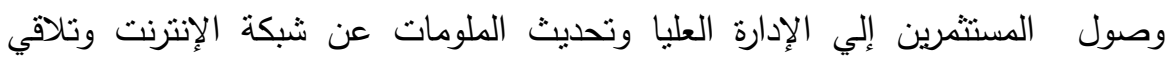

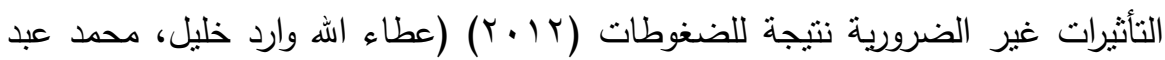

$$
\text { الفتاح لعشماوي). }
$$


r-مجلس الإدارة: مجلس الإدارة هو الذي نوكل لسلطة الإدارة اليومية لأعمال الثركة بالإضافة إلي الرقابة علي الأداء، كما يقوم مجلس الإدارة برسم السياسات العامة للشركة

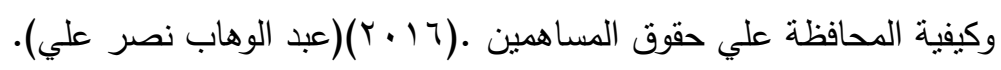

ع-مفهوم إدارة المخاطر في ظل الحوكمة: وتعتبر إدارة المخاطر جزء أساسي من حوكمة الثركات، والتي يمكن تعريفها بأنها " النظام الذي ينت من خلاله نوجيه أنشطة

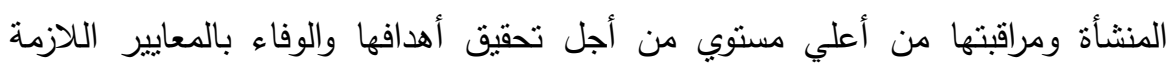

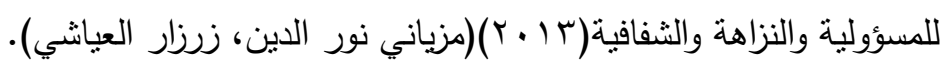

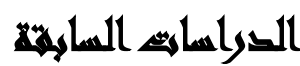

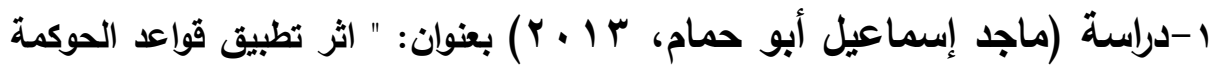
على الإفصاح المحاسبي وجودة التقارير المالية "دراسة ميدانية على الثركات المدرجة لماته

\section{فى سوق فلسطين للأولق المالية "}

تناولت هذه الدراسة بالتحليل والمناقتنة أثر تطبيق قواعد الحوكمة على الإفصاح المحاسبي وجودة التقارير المالية، دراسة ميدانية على الثركات المدرجة في سوق فلسطين

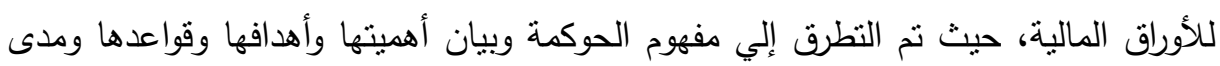

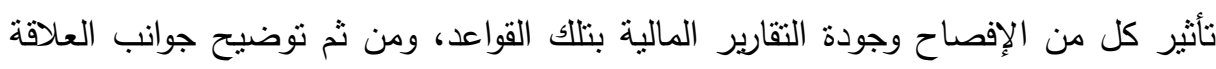
المتداخلة بينهما، وقد استخدم الباحث المنهج الوصفي التحليلي في إجراء الدراسة من خلاد

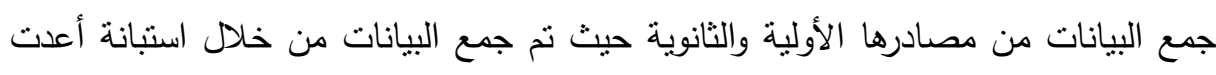

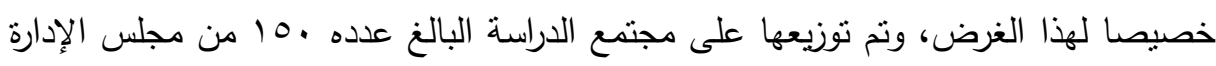
والمديرين التتفيذيين في الثركات المساهمة المدرجة في سوق فلسطين للأوراق المالية العاملة

$$
\text { في فلسطين. }
$$

وتوصلت الدراسة إلى ضرورة تعديق المفاهيم والإلتزام بالقواعد التي قضت بها منظمة

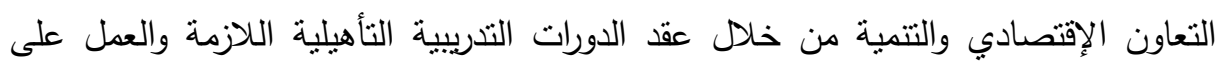

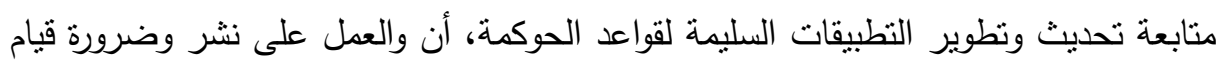

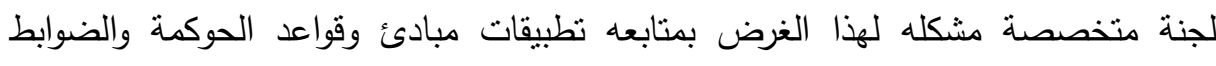

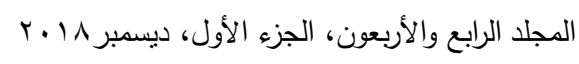


المرتبطة مفهوم وثقافة الحوكمة بشكل أوسع لدى كافة الأطراف ذات العلاقة، وإصدار النشرات والتعليمات التي تعكس ذللك الأمر ، والإلتزام بقواعدها ومبادئها في المجتمع المالي. r-دراسة (حسين عبد الجليل آل غزوي، 1 1 ـ ب) بعنوان: "حوكمة الثركات وأثرها علي مستوي الافصاح المحاسبي في عدم تماثل المعلومات المحاسبية (دراسة اختبارية

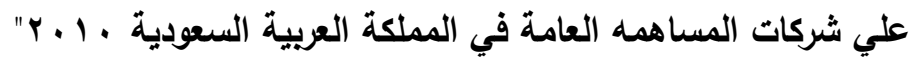
تناولت هذه الاراسة بيان أثر حوكمة الثركات علي مستوي الإفصاح في القوائم المالية لشركات المساهمه العامة في المملكة العربية السعودية. ولتحقيق هذا الهدف لجمع ومعالجة وتحليل 19 شركة كساهمة في المملكة العربية السعودية وذلك عبر مؤشر الافصاح في القوائم المالية وعدم تمانل المعلومات والتي تعتبر أداة القياس لنموذج الدراسة، فتوصلت الدراسة إلي النتائج: وأعلي مستوبي أفصاح في القوائم المالية هو لقطاع الخدمات.

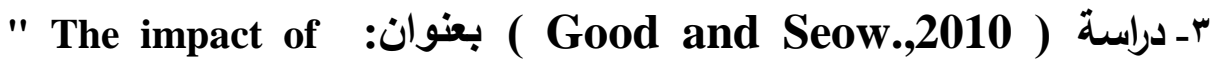
corporate governance on the quality of financial reports and the role of auditors and managers in applying to economic units in

"Singapore

تستعرض هذه الدراسة تأثير حوكمة الثركات علي جودة التقارير المالية ودور المراجعين والمديرين في ذللك بالنطبيق علي الوحدات الاقتصادية في سنغافورة ، وقد خلصت الدراسة إلي التأكيد علي دور المراجعة الداخلية ولجان المراجعة في عملية الحوكمة وفي تحقيق جودة التئي التقارير المالية بالإضافة إلي أهمية القواعد الأخلاقية في ذلك.

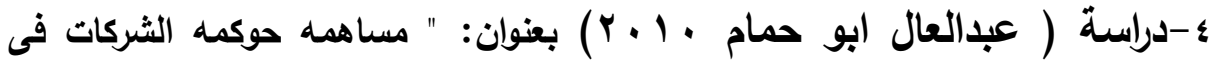

تحقيق جودة المعلومات المحاسبيه والإفصاح المحاسبى لدى المؤسسات الصناعيه " تهاف هذه الاراسة إلى صياغه الجوانب الفكريه لحوكمه الثركات والمفاهيم المتعلقه بها إضافه إلى تسليط الضوء على جوده المعلومه المحاسبيه ومنطلبات تحقيقها والإفصاح المحاسبى وعلاقته بسلامه التقارير الماليه وكذا إستعراض العلاقه المتداخله بين حوكمه

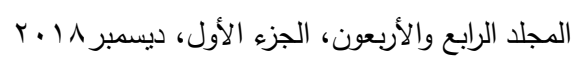


الثركات وجوده المعلومه المحاسبيه بين حوكمه الثركات وجودة المعلومه المحاسبيه والإفصاح المحاسبى ـ وتوصلت الدراسة إلى النتائج التالية: اـ تساهم حوكمه الثركات فى تحقيق جوده المعلومة المحاسبية وذللك من خلال الإعتماد

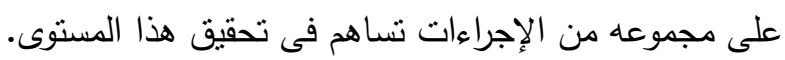
rـ لاتؤدى حوكمة الثركات إلى تسهيل عملية الإفصاح المحاسبى. واقترحت الدراسة على عدة توصيات منها ضرورة العمل على زياده الاهتمام بوظيفه

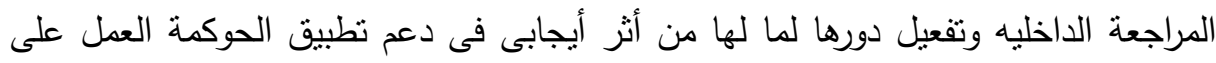
تحقيق مستوى مناسب من الإفصاح فى التقارير الماليه واتاحتها لجميع اصحاب المصالح. وهناك المزيد من الدراسات التي تم عرضها في مثن الرسالة.

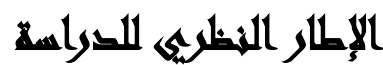

التطور التاريخي لحوكمة الشركات ودوافع ظهورها: نشأ مفهوم حوكمة الشركات بعد ظهور نظرية الوكالة وما تتضمنه من تعارض في المصالح بين إدارة الثركة والمساهمين

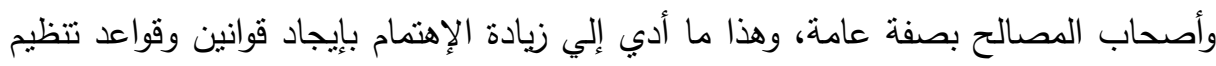
العلاقة بين الأطراف في الشركات.

إن الأساس النظري والتاريخي لحوكمة الثركات يعود لنظرية الوكالة ويعود ظهوره أولاً

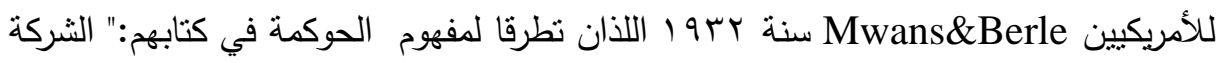

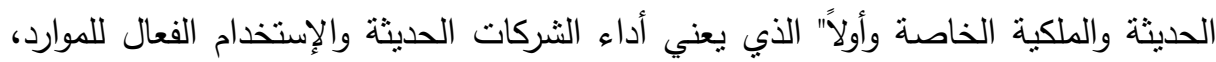
فضلاً علي القضايا المرتبطة بفضل الملكية عن الإدارة.(1) • (1) طالب علاء فرحان). المفهوم اللغوي لحوكمة الشركات: يثير لفظ الحوكمة إلي الترجمة العربية للأصل

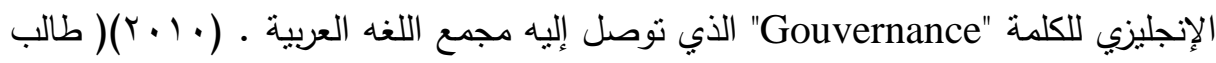

$$
\text { علاء فرحان) }
$$

$$
\text { - ومصطلح الحوكمة كمفهوم يتضمن العديد من الجوانب وهي كالآتي: }
$$


- الحكم: ما يقتضيه من السيطرة علي الأمور بوضع الضوابط والقيود التي تتحكم في

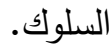

- الاحتكام: ما يقتضية من الرجوع إلأي مرجعيات أخلاقية وثقافية وإلي خبرات، ومن ثم الحصول عليه من خلال تجارب سابقة.

- التحاكم: طلبا للعدالة خاصة من انحراف السلطة وتلاعبها بمصالح المساهمين. كما تعني أيضاً استقلالية سلطة الموافقة والإقرار ومنح التراخيص وشهادادات التهات الإبراء

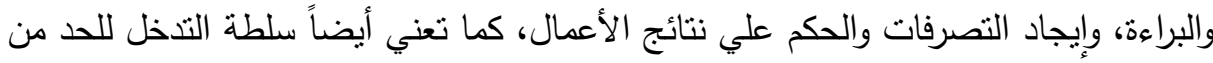

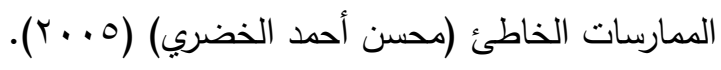

خصائص آليات حوكمة الشركات ومرتكزتها:

تتمثل خصائص الحوكمة في: الإنضباط ،الثفافية،الاستقلالية، المسؤولية، العدالة، والمسؤولية

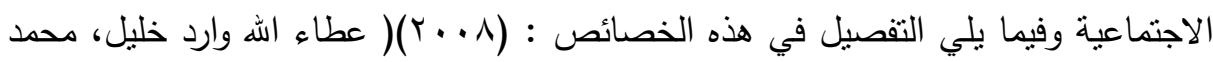
عبد الفتاح لعشماوي) أ- الانضبطي: من خلال توريد بيانات واضحة للجمهور ، ووجود حافز لدي الإدارة اتجاه تحقيق سعر عادل للسهم، والتقدير السليم لحقوق الملكية بالإضافة إلي استخدام الديون في

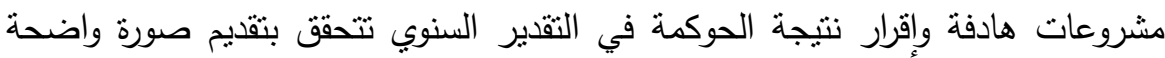
وحقيقية.

ب- الإفصاح والثفافية: ينم ذلك من خلا الإفصاح عن الأهداف المالية بدقة، نشر الثقرير السنوي في موعده نشر النقارير المالية السنوية البيئية في الوقت المناسب، الإهن الإفصاح

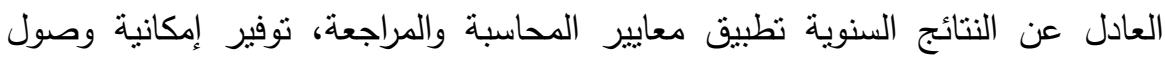

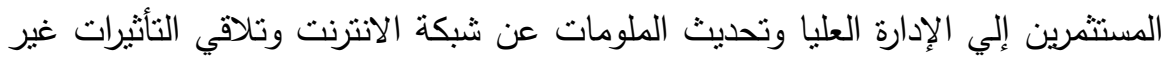
الضرورية نتيجة للضغوطات.

ج- الاستقلالبة: ويتحقق ذلك من خلال المعاملة العادلة للمساهمين من قبل مجلس الإدارة والإدارة العليا وجود رئيس مجلس الإدارة منسق عن الإدارة، وجود لجنة لتحديد المرتبات 
والمكافآت يرأسها عضو مجلس إدارة مستقل، تدعيم وجود مراجعين مستقلين، بمعني إمكانية تقييم وتقدير أعمال مجلس الإدارة.

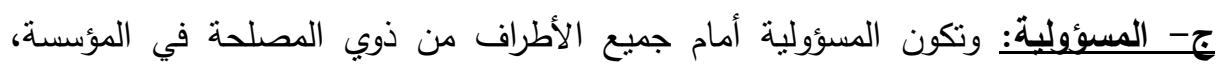
ويتحقق ذلك من خلال عدم قيام مجلس الإدارة الإشراف بدور تتفيذي، وجود أعضاء لمجلس الإدارة مستقلين ومن غير الموظفين، الاجتماعات الدولية والكاملة لمجلس الإدارة.

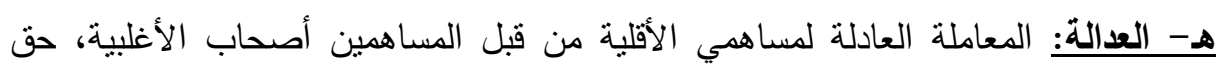

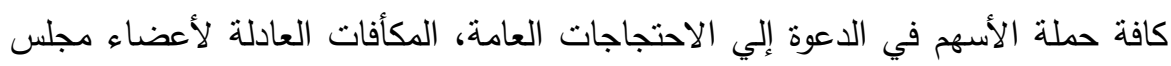

الإدارة.

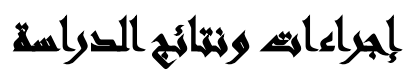

الإجراءات المنهجية للاراسة: إعند الباحث علي المنهج الإستقرائي الإسنتباطي للقراءات

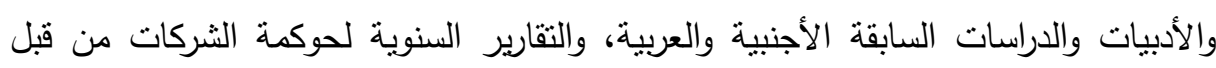

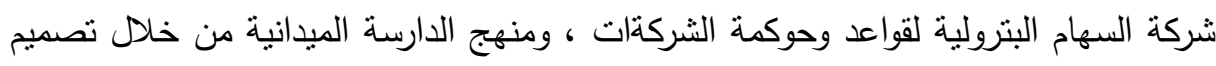
قائمة استقصاء تخص المدراء الماليين والمحاسبين في الثركة، وذلك لمحاولة تحديد أهم

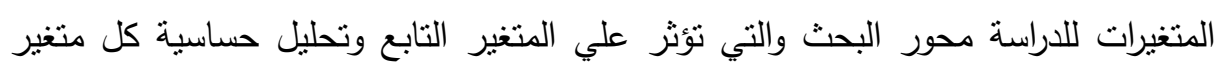
بالنسبة لقياس درجة آليات حوكمة الثركات.

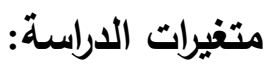
المتغير المستقل: آليات حوكمة الثركات المتغير التابع: مخاطر عدم تماتل المعلومات المات

استخدم الباحث استمارات الإستقصاء كأداة للقياس وأجريت الدراسة علي علئ عينه الدراسة

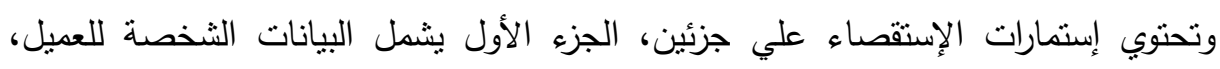
والجزء الثاني يشمل محاورين كما يلي: 1-نطبيق آليات حوكمة الثركات في شركات البنرول بدور لجنة المراجعة ( بتضمن 11 عبارة). r-نطبيق تخفيض مخاطر عدم تماتل المعلومات بدور إدارة المخاطر ( ويتضمن · اعبارة). 
وتتم الإجابة على الفقرات وفق مقياس ليكرت التدريجي المكون من خمس نقاط تتراوح

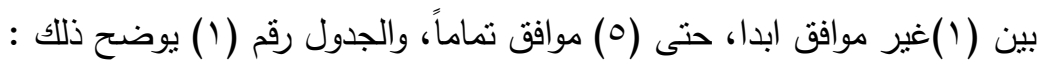
جدول رقم( ( ): يوضح مقياس ليكرت الخماسي

\begin{tabular}{|c|c|}
\hline 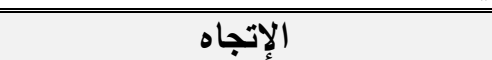 & الفئة \\
\hline تميل الإجابات إلى (غبر موافق أبدا) & $1,69-16 \ldots$ \\
\hline تميل الإجابات إلى (غير موافق) & r.09-1.1. \\
\hline تميل الإجابات إلى (محايد) & $r_{6} r_{9} 9-r_{67}$. \\
\hline تميل الإجابات إلى (موافق) & $\varepsilon_{6} 19-r_{6} \varepsilon \cdot$ \\
\hline تميل الإجابات إلى (مؤافق تماما) & $\varepsilon_{6} Y_{.}$ \\
\hline
\end{tabular}

المصدر: لرينسس ليكرت (2013 ) Likert, Rensis

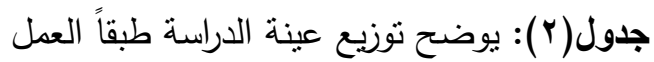

\begin{tabular}{|c|c|c|}
\hline$\%$ & |عدد الاستمارات الموزعه & العمل \\
\hline$r q_{6 .}$ & rᄉ & إدارة عليا (مدير تتفيذى) \\
\hline 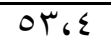 & $V$. & مراقب حسابات \\
\hline $1 V_{6} 7$ & TM & مراجع داخلي \\
\hline $1 \cdots \cdot 6$ & 111 & الاجمالى \\
\hline
\end{tabular}

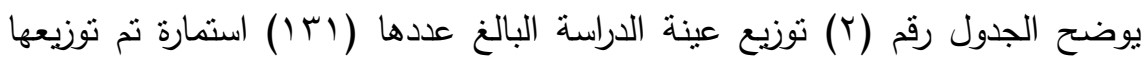

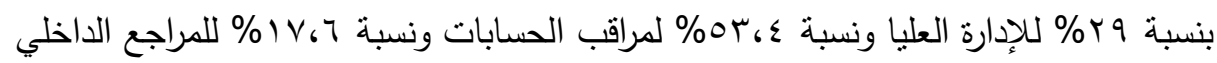
الاختبارات الإحصائية المستخدمة: ا-إختبار لإستمارة الإستقصاء: إختبار ألفاكرونباخ Cronbac لقياس ثبات وصدق محتوى استمارة الإستقصاء الدراسة. حيث يوضح مدى إمكانية الاعتمادية على نتائج قائمة الإستقصاء، ومدى إمكانية تعميم

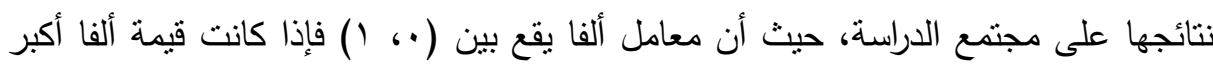
من أو تساوى 7، . أمكن الإعتماد على عينة البحث وتعميم نتائجها على مجتمع الدراسة. 
جدول رقم(ץ): يوضح درجات مقياس ألفا كرونباخ Cronbach's alpha درجئ

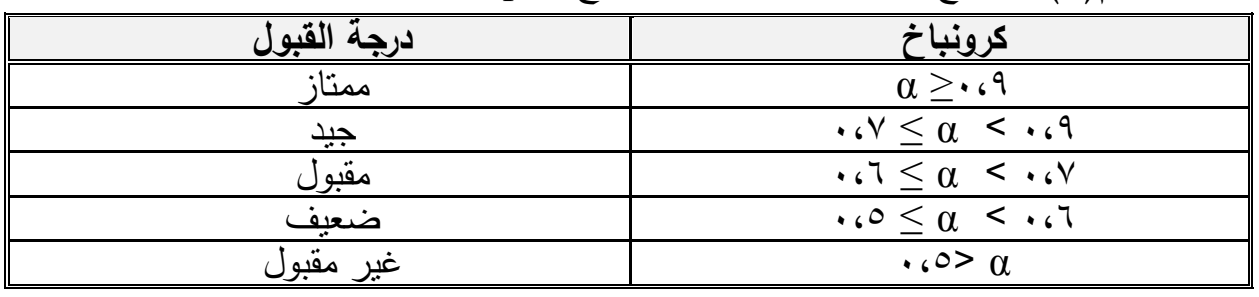

r-الإختبارات الإحصائية تضمنت:

• المتوسط والانحراف المعياري ومعامل الإختلاف ونسبة الإتفاق لمعرفة اتجاه آراء عينة الدراسة • اختبار فريد مان Friedman Test لمعرفة مدى الإتفاق والإختلاف بين آراء عينة الدراسة حول الأهمية النسبية للعبارات.

• معامل الإرتباط البسيط لبيرسون Pearson Correlation لمعرفة العلاقة بين متغيرات الدراسة • تحليل الإنحدار البسيط والمتعدد المتدرج لمعرفة آثر المتغيرات المستقلة على المتغير التابع لئن وللتحقق من صحة فروض الدراسة. صدق وثبات درجات الأداة: ا. صدق الأداة: تعتبر الأداة صادقة إن كانت تقبس ما وضعت لقياسه، أو الصفة التي تهدف إلى قياسها، فإذا كانت أداة الدراسة تقيس اتجاه المبحوثين نحو شيء التهاد معين، فيجب

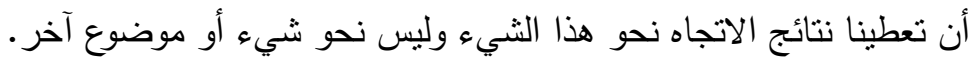

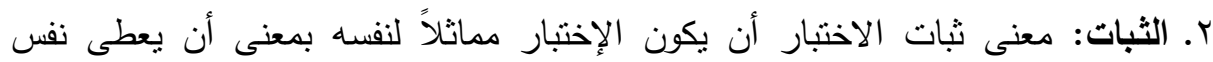

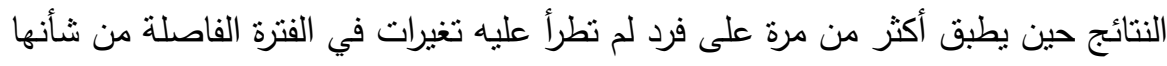

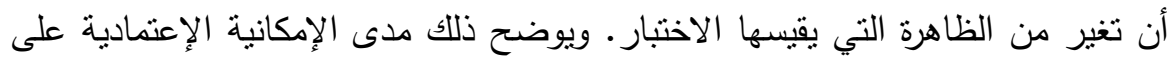

نتائج قائمة الإستبيان، ومدى إمكانية تعميم نتائجها على مجتمع الدراسة.

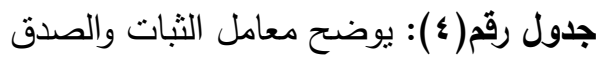

\begin{tabular}{|c|c|c|}
\hline معامل الصدق & معامل الثبات & البيان \\
\hline • ‘ $\wedge V \varepsilon$ & . 6170 & |طبيق آليات حوكمة الثركات في شركات البترول بدور لجنة \\
\hline - ‘110 & • ‘ $\vee \wedge \varepsilon$ & |طبيق تخفيض مخاطر عدم تماتل المعلومات بدور إدارة المخاطر \\
\hline .6940 & $\therefore$ ¿A>0 & الاجمالي \\
\hline
\end{tabular}

Spss المصدر: من مخرجات برنامج

604

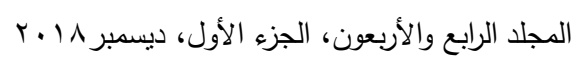


يتضح من الجدول رقم (ع) حين تقترب قيمة معامل الثبات من الواحد الصحيح ثتوافر المصداقية قفي البيانات وكما اقتربت من · . اتكون المصداقية أكبر، حيث أن النتائج المتعلقة بتحليل هذه الدراسة تجاوزت . ب\% وهي قيمة مقبولة إحصائياً كمؤشر في صدق وثبات قائمة الاستقصاء.

\section{التحليل الوصفي:}

اولاً: تطبيق آليات حوكمة الثركات في شركات البترول بدور لجنة المراجعة كما يلي:

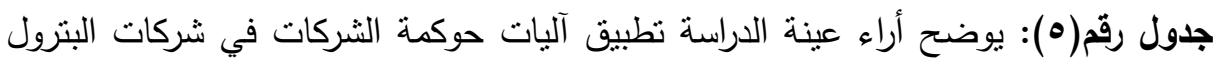

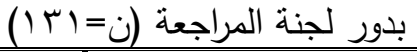

\begin{tabular}{|c|c|c|c|c|c|}
\hline الإتجاه & الموافقة & الإختلاف معامل & الإنحراف & |المتوسطح & طبيعة ونوعية المعلومات \\
\hline ل موافق بشدة & $9 T_{6}$ & 11,0 & $.60 \leqslant$ & $\varepsilon 677$ & تقوم اللجنة بوضع تقرير مكتوب بمالاحظاتها وتوصياتها \\
\hline ل موافق بشدة & 1967 & $1 r_{6}$ & $.67 \%$. & 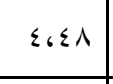 & تقوم اللجنة بدراسة نقارير إدارة المراجعة الداخلية ووضع الاجحراء \\
\hline ل موافق بشدة & $\wedge 9$ ८ & 106 & $.67 \mathrm{~V}$ & $\varepsilon \leqslant \Sigma V$ & تقوم اللجنة بعرض تقرير بنتائج أعمالهم علي مجلس إدرارة الثركة. \\
\hline كوافق بشدة & $\wedge 9$ ، & 10,4 & .671 & $\varepsilon$ ¿ & تختص اللجنة بدراسة هيكل الرقابة الداخلية. \\
\hline ل موافق بشدة & 19, & Ir.V & .671 & $\varepsilon$ \& & قوم اللجنة باعداد تقرير دوري كل ثلاثة أشهر عن نتائج \\
\hline ل موافق بشدة & $\Lambda_{\Lambda} \Lambda_{\mathrm{r}}$ & $1 r_{6} 0$ & .600 & $\varepsilon$ « & 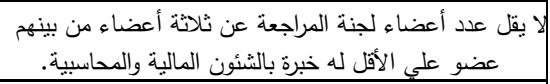 \\
\hline ل موافق بشدة & $\wedge V_{6}$. & 18.9 & $\vee \Lambda_{6}$. & E, ro & تم تتكيل لجنة المراجعة من أعضاء مجلس الإدارة غير \\
\hline ل موافق بشدة & $10, r$ & $\mid V_{6} 1$ & $.6 \mathrm{VT}$ & E, YT & 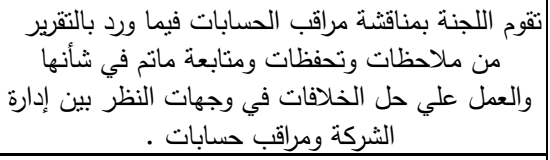 \\
\hline 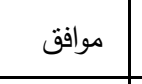 & $\Lambda_{\top} r_{\triangleleft}$ & $1 \leqslant 67$ & .671 & $\varepsilon 619$ & تبدئ اللجنة ملاحظاتها علي مراقب الحسابات في إسناد أية \\
\hline 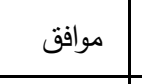 & Ar, & $r \cdot r$ & $\cdot 610$ & $\varepsilon 619$ & 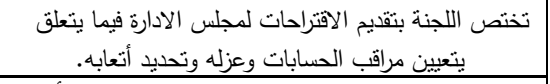 \\
\hline 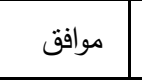 & $\Lambda \Lambda_{6}$ & $1 \leq 67$ & .671 & $\varepsilon 61 V$ & تختص اللجنة بدراسة تقرير مراقب الحسابات بشأن \\
\hline \multicolumn{5}{|c|}{9.674} & قيمة كاr \\
\hline \multicolumn{5}{|c|}{$\cdots \cdots$} & توى الدلالة \\
\hline
\end{tabular}

Spss المصدر : من مخرخرات برنامج

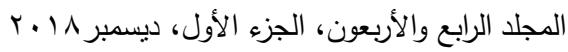


يتضح من بيانات الجدول (0) إلى وجود اختلاف معنوي في الأهمية النسبية لآراء

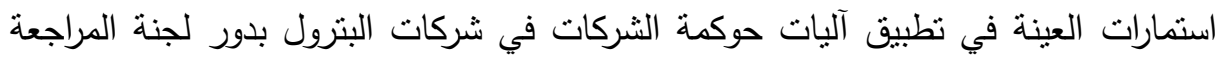

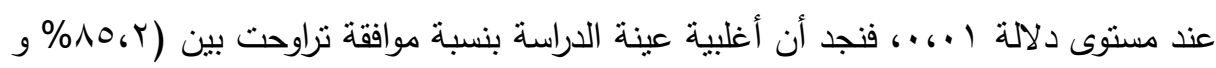

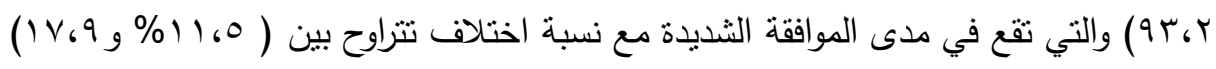

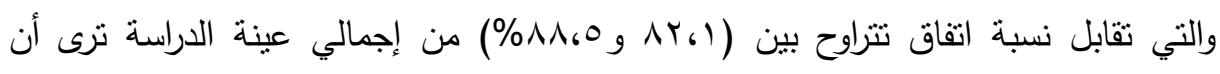

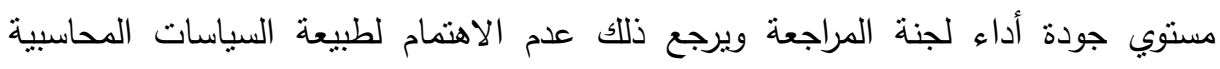

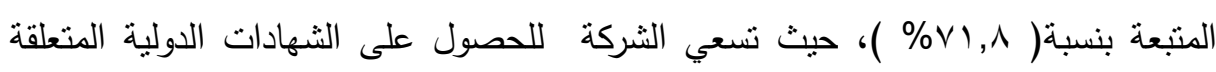
بآليات الحوكمة.

ثانياً: تطبيق تخفيض مخاطر عدم تماثل المطلومات بدور إدارة المخاطر كما يلي:

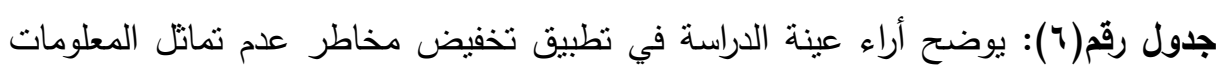

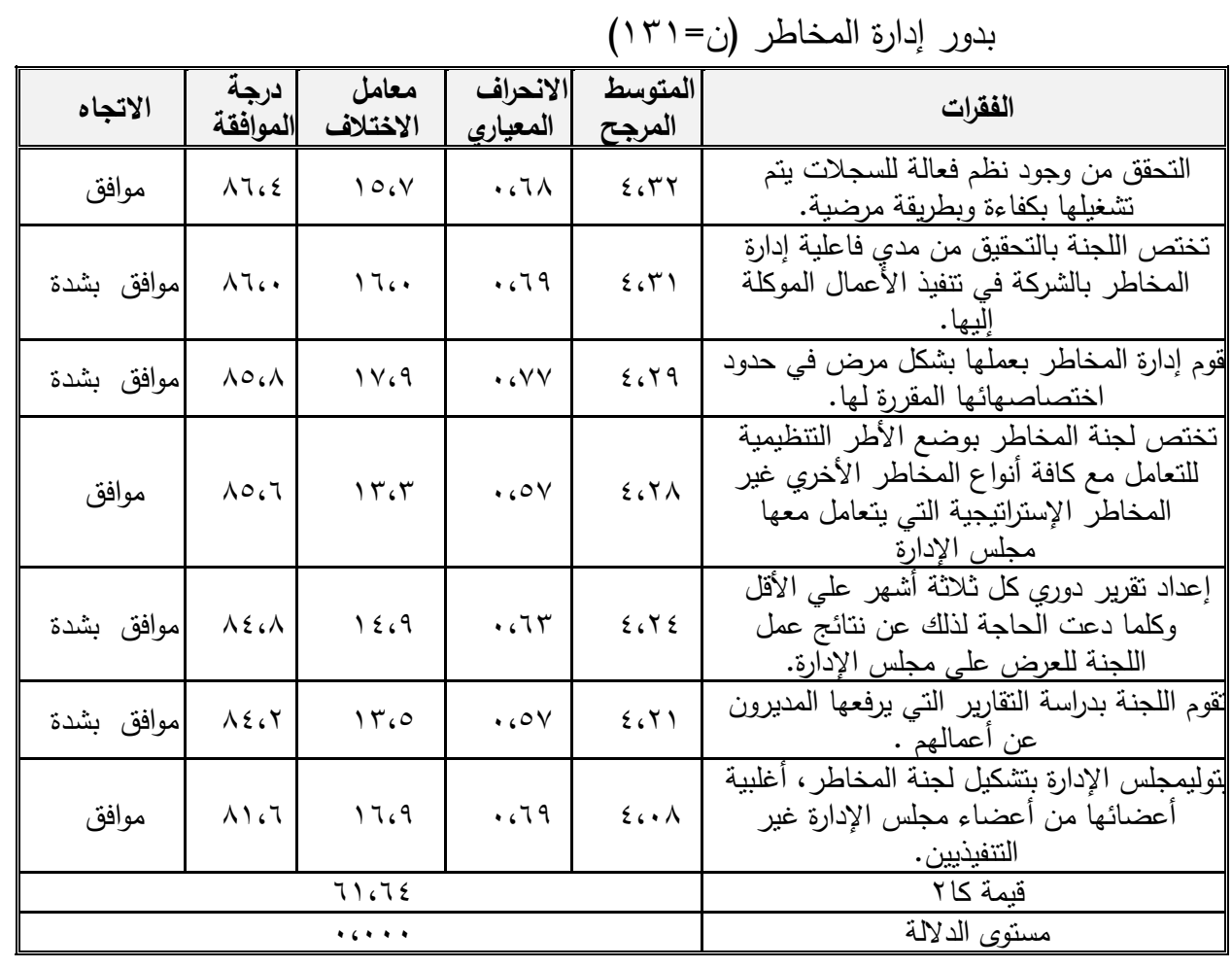

Spss المصدر: من مخرجات برنامج 
يتضح من بيانات الجدول رقم (؟) إلى وجود اختلاف معنوي في الأهمية النسبية لآراء مفردات العينة في الأهمية لفاعلية إدارة المخاطر هي كيفية التحقق من وجود نظم فعالة

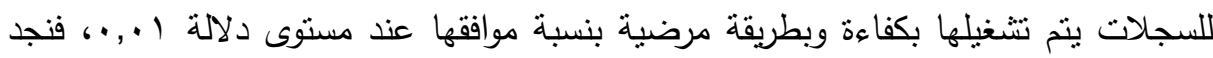

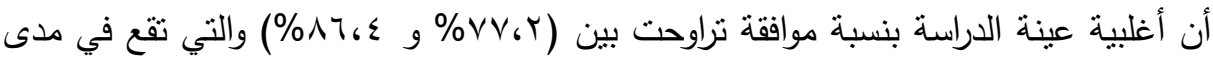

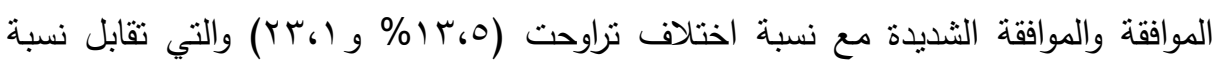

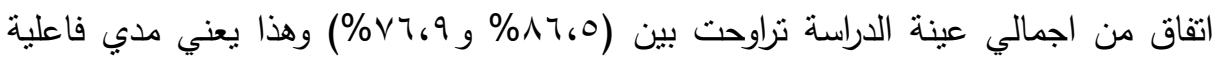
إدارة المخاطر بالثركة في تتفيذ الأعمال الموكلة إليها التي كانت تمثل (

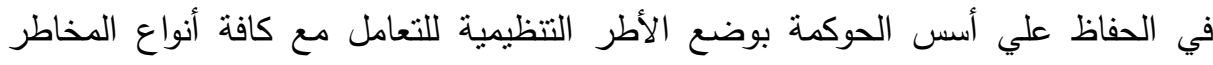
الأخري غير المخاطر الاستراتيجية التي يتعامل معها مجلس الإدارة المتمنلة (ب, •ـم) ويليها

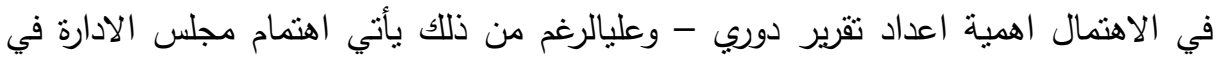

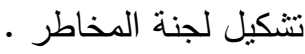

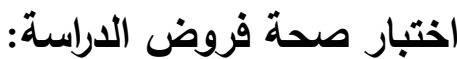

الفرض الأول: توجد علاقة ذات دلاله إحصائية نحو أثر تطبيق آليات حوكمة الشركات وتخفيض مخاطر عدم تماثل المعلومات. الفرض الثاني: نوجد علاقة ذات دلاله إحصائية نحو أثر تطبيق آليات حوكمة الثركات علي

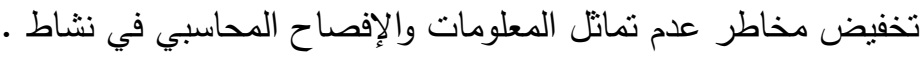
لإختبار صحة فروض الدراسة تم استخدام معامل الإرتباط البسيط وتحليل الإنحدار

$$
\text { البسيط والجدول رقم (^) يوضح ما يلي : }
$$

المتغير المستقل: آليات حوكمة الشركات

\begin{tabular}{|c|c|c|c|c|c|c|c|c|}
\hline مستوى الدلالة & فيمة & التحديبي & معامل & الارتباط & 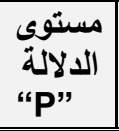 & "يمة تئئ & المعلمة & \\
\hline \multirow[b]{2}{*}{$\cdot 6 \cdot 1$} & \multirow[b]{2}{*}{ r r.AV } & \multirow[b]{2}{*}{$1 \leq 67$} & \multirow[b]{2}{*}{$.610,4$} & \multirow[b]{2}{*}{.6491} & .6 .1 & $\left.\Lambda_{6}\right) \leqslant 7$ & \%०,7.7 & الثابت \\
\hline & & & & & $\cdot 6.1$ & 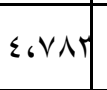 & $.6 \leq T \leq$ & آليات حوكمة الشركات \\
\hline
\end{tabular}


يتضح من نتائج الجدول رقم (^) وجود علاقة إرتباطية طردية بين آليات حوكمة

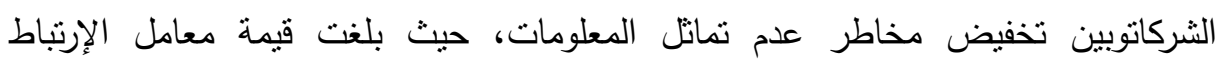

..6

القرار صحة فرض الدراسة: نوجد علاقة ذات دلاله إحصائية نحو أثز تطبيق آليات حوكمة الشركات وتخفيض مخاطر عدم تمانل المعلومات. صحة فرض الاراسة الثاني: توجد علافة ذات دلاله إحصائية نحو أثز تطبيق آليات حوكمة

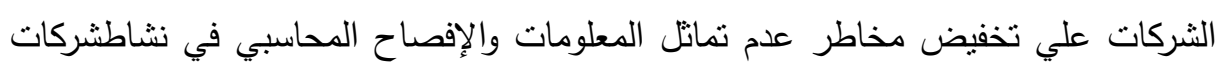
البترول.

المتغير التابع: مخاطر عدم تماثل المعلومات المتغير المستقل: آليات حوكمة الشركات

جدول رقم(9): قيمة معامل الإرتباط ومستوى الدلالة بين المتغير التابع والمتغيرات المستقلة

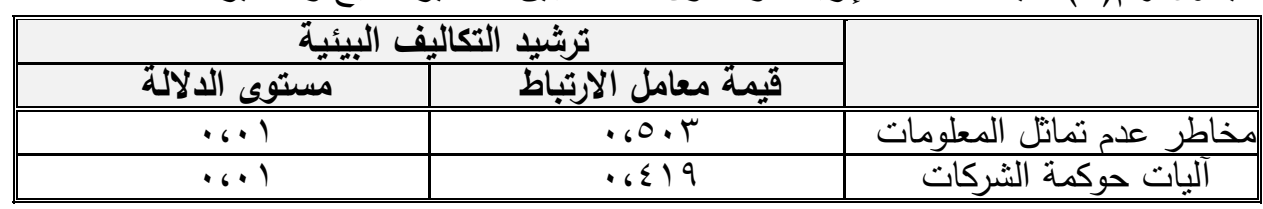

\begin{tabular}{|c|c|c|c|c|c|c|c|c|}
\hline & & & & & الانحد & تائج تحا & ): يوضح & جدول رقم \\
\hline الدلالة & قيمة ف & التحاليد & معامل & $\begin{array}{c}\text { الإتباط } \\
\text { الكعاملي } \\
\end{array}$ & $\begin{array}{l}\text { الدلالدة } \\
\text { "P" } \\
\end{array}$ & قيمة ت "t" & المعلمة & \\
\hline \multirow{3}{*}{$\cdots$} & \multirow{3}{*}{ Y4. } & \multirow{3}{*}{. GrA. } & \multirow{3}{*}{. } & \multirow{3}{*}{$.0 \leqslant}$. & 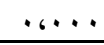 & $96 .+99$ & $r 7,101$ & الثابت \\
\hline & & & & & $\cdots \cdots$ & £.07\& & דודי. & مخاطر عدم المعلومات \\
\hline & & & & & .61. & T,TIT & .6171 & اليات حوكمة \\
\hline
\end{tabular}

يتضح من نتائج الجدول رقم (· (1) وجود علاقة ارتباطية طردية بين مخاطر عدم تماتل المعلومات البيئية من آليات حوكمة الثركات حيث بلغت قيمة معامل الإرتباط على الترتيب . . 0. . . . . 6 919 
القــرار صحة فرض الدراسة: توجد علاقة ذات دلاله إحصائية نحو أثر تطبيق آليات حوكمة الثركات علي تخفيض مخاطر عدم تمانل المعلومات والإفصاح المحاسبي في نشاط الثركة.

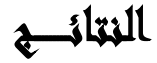 \\ أولاً: أهم النتائج التي توصلت إليها الدراسة التي اجراها الباحث كما يلي:} 1-يعد تخفيض مخاطر عدم تماتل المعلومات وظاهرة حوكمة الثركات وجهان لعملة واحدة، يؤثر كل منهما في الآخر يتأثر به، فتخفيض مخاطر عدم تماتل المعلومات في ظل آليات حوكمة الثركات تصبح أكثر شفافية للثركة وتعزز مصداقية المعلومات المحاسبية. r-تلعب آليات حوكمة الشركات درواً كبيراً في إدارة الأزمات، فهي تعتبر بمثنابة الأداة التي

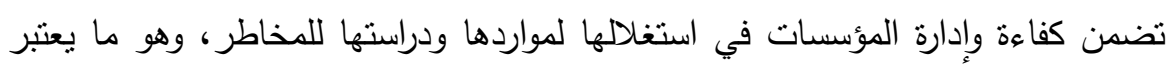
كمؤشر عن تحقيق الثركة لأهدافها بالدرجة الأولي.

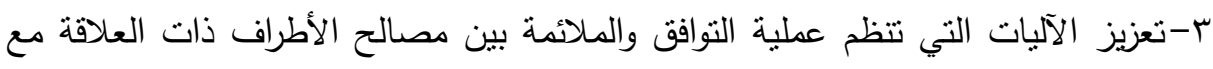
الثركات.

ثانياً: نتائج خاصة بصحة فروض الاراسة: قامت الدراسة على فرضين رئيسيين حيث أنتبت

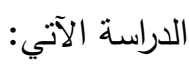
قبول الفرض: توجد علاقة ذات دلاله إحصائية نحو أثر تطبيق آليات حوكمة الثركات وتخفيض مخاطر عدم تماتل المعلومات. قبول الفرض: نوجد علاقة ذات دلاله إحصائية نحو أثر تطبيق آليات حوكمة الثركات والإفصاح المحاسبي في نشاط الشركة.

\section{المهروسيامت}

في ضوء نتائج الدراسة يوصي الباحث بما يلي: 1-إجراء دراسة مستقبلية عن تأثثر دور مجلس الأدارى علي جودة التقارير والقوائم المالية للتحقيق والتأكد من عدم وجود تلاعب بالتقارير والقوائم المالية.

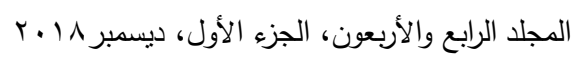


r-نشر ثقافة آليات حوكمة الثركات في المجتمعات عن طريق وسائل الإعلام ومنظمات

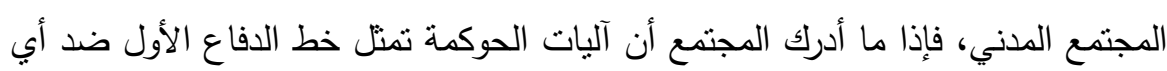

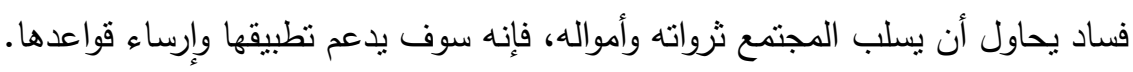

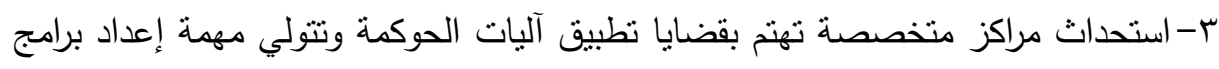
تدريبية لترسيخ أهداف ومنطلبات الحوكمة علي تخفيض مخاطر عدم تمانل المعلومات في

$$
\text { الشركات. }
$$

ع- العمل المستمر علي تطوير مفهوم وقواعد آليات الحوكمة التنفيذية من الناحية المهنية. ه- إصدار قوانين تتص علي نطبيق مبادئ حوكمة الثركات بالنسبة لتخفيض مخاطر عدم عند تمنل المعلومات من أجل تفعيل وتطوير شركات البترول.

\section{enll}

سمر أحمد السيد(10 • ب): " قياس تطبيق تأثنر آليات الحوكمة علي زيادة القدرة التتبؤية

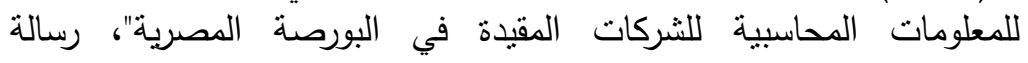

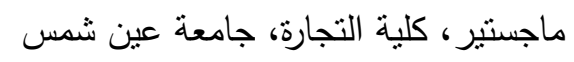

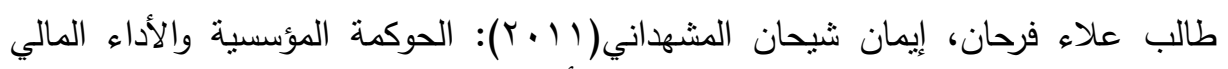

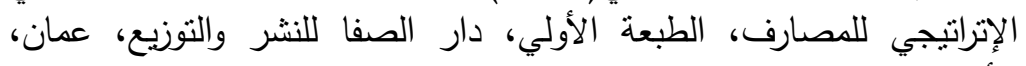

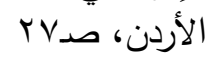

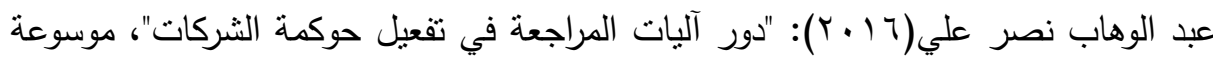

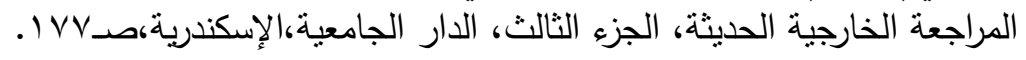

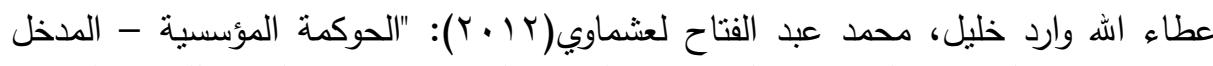

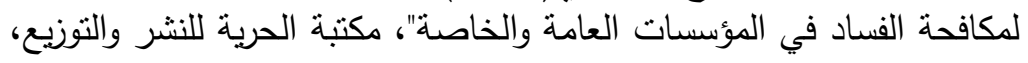

$$
\text { صدr }
$$

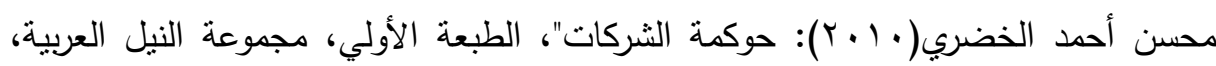

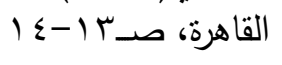

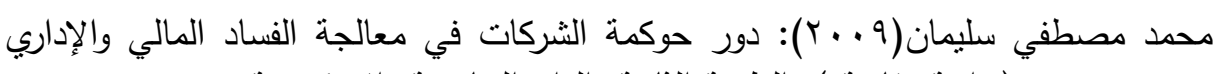

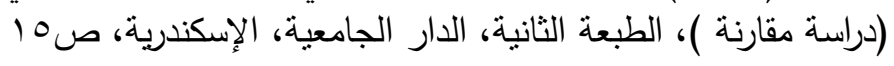




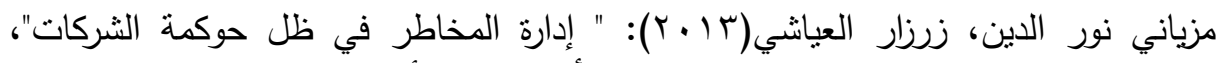

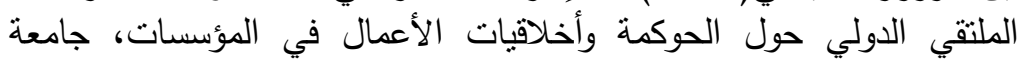

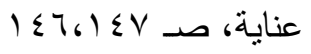

Alain Fient et autre , (2015): Gouvernement d'entprise, deboech, paris,pp.38 .

Haward p.slettler, (2008): "audit principles methods generals", edotoin francaise (clm pibli-union),paris, ,pp.56-57.

Helene PLOIX.Le dirigeant et gouvernement d'entreprise,(Paris:Village Mondial,2010,pp.20.

Michael Elliott, et al, an improcess model for internal auditing, managerial auditing journal.vol.22,2015,pp552-565.

Makram Chemangui,"Proposition d'une metriaue la qualite de l'audit:experimentation dans le cadre des relations d'agence interne ",la revue de l'association francophone de comptabilite, tome 15,vol.1,2010,p.228. 
مصطفي محمود سليم عبد الكريم وآخرون

\title{
APROPOSED FRAMEWORK FOR APPLYING MECHANISMS OF CORPORATE GOVERNANCE ON REDUCING RISKS OF INFORMATION ASYMMETRY IN OIL COMPANIES CASE STUDY
}

\author{
Abd-Elkareem, M. M. S. ${ }^{(1)}$;Mosa, Smasm, K. ${ }^{(2)}$ \\ and Alahmady, B. M. ${ }^{(2)}$ \\ 1) Institute of Environmental Studies and Research, Ain Shams \\ University 2) Faculty of Commerce, Ain Shams University
}

\begin{abstract}
This study aimed at identifying the extent to which corporate governance mechanisms are applied to reduce the risks of information asymmetry in oil companies , By applying a case study to an oil company.

To achieve this goal, the researcher relied on theoretical rooting through the inductive method, Of books and references and studies of Arabic and foreign, And then conduct a case study on the oil arrows company through the design of a survey list of accountants and auditors and financial managers and executives in the company mentioned high, The sample of the study was represented by the Petroleum Arrows Company. The forms were distributed at the levels of the financial managers and accountants for 131 and 131 assemblies at the levels mentioned in the petroleum arrows company. The study concluded that there is a significant and significant correlation between the effect of applying the corporate governance mechanisms and reducing the risks of information asymmetry, It also recommended the establishment of specialized centers concerned with the implementation of governance mechanisms and the task of preparing training programs to consolidate the objectives and requirements of governance to reduce the risks of information asymmetries in companies.
\end{abstract}

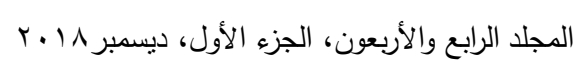

